\title{
Stochastic physics, complex systems and biology
}

\author{
Hong Qian* \\ Department of Applied Mathematics, University of Washington, Seattle, WA 98195, USA \\ * Correspondence: hqian@u.washington.edu
}

Received September 30, 2012; Revised November 18, 2012; Accepted December 7, 2012

\begin{abstract}
In complex systems, the interplay between nonlinear and stochastic dynamics, e.g., J. Monod's necessity and chance, gives rise to an evolutionary process in Darwinian sense, in terms of discrete jumps among attractors, with punctuated equilibria, spontaneous random "mutations" and "adaptations". On an evolutionary time scale it produces sustainable diversity among individuals in a homogeneous population rather than convergence as usually predicted by a deterministic dynamics. The emergent discrete states in such a system, i.e., attractors, have natural robustness against both internal and external perturbations. Phenotypic states of a biological cell, a mesoscopic nonlinear stochastic open biochemical system, could be understood through such a perspective.
\end{abstract}

\section{INTRODUCTION}

Biological systems and processes are complex. One of the hallmarks of complex behavior is uncertainties, either in the causes of an occurred event, or in predicting its future $[1,2]$. This "feel" of complexity is intimately related to the following issue [3]: When a system consists of only a few degrees of freedom, say $x_{1}, x_{2}$ and $x_{3}$, a complete description of the "trajectory" of $\left(x_{1}, x_{2}, x_{3}\right)(t)$ for all $t$ constitutes a full understanding of the system. However, when a system has a million of degrees of freedom, $\boldsymbol{x}(t)=\left\{x_{i}(t) \mid 1 \leqslant i \leqslant 10^{6}\right\}$, a complete description of the $\boldsymbol{x}(t)$ is not informative at all! One needs to find a particular "angle" to synthesize the large amount of data, or a "pattern" to obtain a summary. In classical physics of inanimate matters with relatively homogeneous individuals, this is accomplished by introducing the concept of distribution together with macroscopic thermodynamic quantities, giving rise to the discipline of statistical thermodynamics. In modern cellular biology, this is known as "data interpretation with respect to biological functions": Usually a narrative in addition to the data is required [4].

The foregoing brief discussion points to a key departure from the classical physics of Newton and Laplace [5]: A rational choice of mathematical descriptions of biological systems and processes requires a probabilistic view of the dynamics, which provides both individual-based and population-based perspectives. Studying system dynamics in terms of stochastics, either due to intrinsic uncertainties, lack of full knowledge, or due to a need for organizing large amount of data, is the basis of what we call stochastic physics.

\section{WHAT IS STOCHASTIC PHYSICS}

Modern sciences emphasize quantitative representation of experimental observations, widely known as mathematical modeling. Along this line, there are two types of modeling: the data-driven and the mechanism-based models. In the history of physics, Kepler's model (laws) was the most celebrated example of the former, while Newton's theory of universal gravity, which "explains" Kepler's results, is the canonical example of a mechanism. In fact, the very term mechanism was derived from the word mechanics. In biology, Mendel's model (law) was the former, and Hardy-Weinberg's theory was the latter. The difference between the examples in physics and the examples in biology is that the latter have to take into account of uncertainties. Data-driven modeling incorpor-

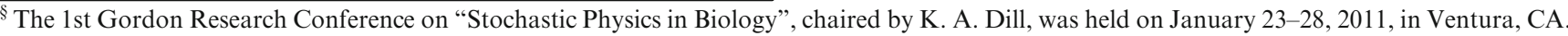


ating uncertainties gives rise to the entire field of statistics - and bioinformatics and financial engineering are two most active branches of studies in recent years.

This leads straight to the question "where is the mechanism based modeling with uncertainty"? Stochastic physics is precisely the answer to this calling. In sociology and economics, this type of modeling is called agent-based, and in finance it is called behavior finance.

In applied mathematics, statistics is associated with data-driven modeling and stochastic process is associated with population distribution based mechanistic modeling. In physics, statistical physics has traditionally dealt with more on state of matters in equilibrium rather than dynamics of open, driven systems. Nevertheless, it is a shining example of successful stochastic modeling.

\section{NONLINEAR PHYSICS AND STOCHASTIC PHYSICS}

Stochastic physics shares many of the concepts and concerns of the nonlinear physics that has gone before it: They both are focused on dynamics of a system [6]. Technically, for nonlinear systems exhibiting chaotic dynamics, a characterization based on distribution turns out to be more appropriate [7]. Data analyses of chaotic signals also constantly employ methods from statistics $[8,9]$.

Stochastic dynamics in linear systems and nonlinear systems are fundamentally different $[10,11]$. The former can be essentially represented by a Gaussian process, which was extensively studied by eminent physicists like Uhlenbeck, Chandrasekhar, and Onsager [12-14]. But stochastic dynamics per se is not the reason for complex behavior. A Gaussian process has certain unpredictability, nevertheless the ultimate fate of the dynamics is all the same: It fluctuates around its mean value.

However, when one faces a strongly nonlinear system with stochasticity, one has to talk about evolution, evolution process in Darwin's sense with punctuated equilibria and spontaneous random "mutations" and "adaptations". This is one of the profound insights derived from the studies of nonlinear stochastic systems: The fluctuations in a nonlinear system with multiple attractors make rare events, something with infinitesimal probability from a deterministic stand point, an sure occurrence with probability one in an "evolutionary" time scale $[15,16]$. This picture fits J. Monod's notion of chance and necessity $[6,17]$. Furthermore, when encountering external environmental changes, nonlinear multistable systems exhibit adaptation by enhanced rate of transition into the "favored attractors"; and ultimately exhibit "rupture" - the nonlinear catastrophe scenario in the presence of stochasticity [18].
Newton-Laplace's dynamics gives us a sense of convergence. For strongly nonlinear stochastic dynamics, the validity of the converging dynamics is only on a rather limited time scale. In an evolutionary time scale, divergent dynamics emerges. This, we believe, is a philosophical implication derived from stochastic physics [5].

\section{STOCHASTIC PHYSICS AND QUANTITATIVE BIOLOGY}

Physics and computer science (CS) are two cornerstones of modern, engineering world. Therefore, it is not surprising that they support the most quantitative aspects of biology. Yet, upon a more careful reflection, one realizes that thinkings in both physics and CS are in odd with that of biologists: Physics considers systems that can be described with a few variables, known as "information poor" according to Hopfield [3], and CS, while deals with much more complex problems, nevertheless in terms of perfect logics with almost infinite precision. Biological systems are information rich, and biological processes are not about precision or optimal, but rather about functional and survival.

The studies of biological cells, the universal building block of living organisms, also have two foundations that echoed physics and CS: biochemistry and genomics. Biochemistry is founded on the tradition of physics, via the investigations of macromolecular structures and dynamics and biochemical reactions, while genomics heavily utilizes concepts and methods from CS, i.e., coding, information, discrete mathematics, leading to the emergence of bioinformatics in recent years. The heavy influences of physics and CS in biological thinking, in fact in all 21-century modern thinkings, is unmistakable. Nowadays, even the studies of biochemical reaction systems are usually about their information logic flow. Known as signal transduction, it provides a clear link between biochemistry within a cell, to perceived function. However, one often forgets that information is only an abstract term; its physical bases have to be either energy or material. In cell biology, they are represented by the structures and states of macromolecules. The information logic flow aspects of biochemical reaction is our "models" and "interpretations" of a biological organism based on our understanding of its engineering functions! It is a "narrative" cell biologists provide to understand a complex reality [19].

This reveals an important gap in the current dominant thinking of cell biology: the link between the physics of molecules, the chemistry of reactions, and the information logic flow they represents. It is widely recognized that investigations into this link require statistical physics and 
molecular thermodynamics in small systems with dynamics [20-22]. Filling this gap has been called for as the systems biology of cells [23]. Though yet to be proven, it is not difficult to see that the stochastic physics approach as described above has the potential to be a powerful, quantitative language of cellular dynamics and other biological systems [24].

The stochastic physics approach to biology relies more on mechanistic understanding of biological systems and processes than on high-throughput large data sets. It is a powerful tool to generate working hypotheses in a rigorous way. In current biological research, one often states that "we like to know how it works". However, a scientifically more sound statement should be "we like to know whether it works in this way". This goes back to the hypothesis-driven research with strong inference [25]. Taking uncertainties into account, stochastic modeling is based on one's mechanistic understanding, and relies on mathematical deduction to generate precise hypothesis. It will be an indispensible tool in biological research on par with data-driven bioinformatics.

\section{CELLULAR BIOLOGY AND THEORY OF EVOLUTION}

Based on the Modern Synthesis of Darwin's theory of evolution, the current population genetics and genomics [26] attribute the molecular basis of biological variations to different DNA sequences, which is inheritable through Mendelian genetics and Watson-Crick base-pairing mechanism. Biochemistry, however, has been always considered as merely a deterministic mechanics that executes the instructions coded in the DNA [27].

Recent laboratory measurements on stochastic gene expression in single cells with single-molecule sensitivity, however, have broken the genomic monoplay of biological variations [28,29]. Stochasticity has been increasingly recognized as a key aspect of cellular molecular biology. In terms of Darwin's evolution, Kirschner and Gerhart have maintained that the essential role of cellular and organismal biology is to provide phenotypic variations with plausible molecular mechanisms that bridge genomes and lives [30].

The tenets of stochastic physics fit this perspective. In particular, the mathematical theory of stochastic processes has revealed a rich thermodynamic structure in any stochastic dynamics based on Markov formalism [31]. The thermodynamic theory clearly distinguishes a closed stochastic system which reaches an equilibrium distribution with detailed balance, and an open, driven stochastic system which reaches a nonequilibrium steady state [3235]. It has been firmly established that the latter corresponds to precisely cellular biochemical systems upon which continuous chemical driving forces are applied. The conversion of chemical energy into heat in isothermal cellular systems can be characterized by entropy production rate [36,37].

The external energy supply, as the "environment condition" for an open system, is the thermodynamic necessity for self-organization and complex behavior [36]. Thermodynamics, however, can only tell what is possible and what is not; but it does not tell what is feasible and what is the mechanism. For the latter, detailed "molecular mechanisms" have to be developed. There is clearly a dichotomy between the nature vs. nurture for the function of a biochemical system. A stochastic description of dynamics provides a unique tool to understand the occurrence of sequential events, i.e., kinetics, in terms of the "most probable path" [38-40].

There is a growing interest in understanding cell differentiation including stem cell differentiation and reprogramming, isogenetic variations, and even cancer carcinogenesis from an evolutionary perspective at the cellular level $[39,41,42]$. The mathematical theory of evolution and population genetics has long been based on stochastic processes [43-45]. Therefore, the stochastic physics approach to cellular biochemical dynamics provides a natural unifying framework to further this exciting new frontier of biological science.

A stochastic physics based quantitative understanding of cellular biology, in return, will provide a paradigm for studying other complex systems $[11,32,46]$.

\section{REFERENCES}

1. Mackey, M. C. (1989) The dynamic origin of increasing entropy. Rev. Mod. Phys., 61, 981-1015.

2. Ge, H., Pressé, S., Ghosh, K. and Dill, K. A. (2012) Markov processes follow from the principle of maximum caliber. J. Chem. Phys., 136, 064108 .

3. Hopfield, J. J. (1994) Physics, computation, and why biology looks so different? J. Theor. Biol., 171, 53-60.

4. Knight, J. (2002) Physics meets biology: bridging the culture gap. Nature, 419, 244-246.

5. Prigogine, I. and Stengers, I. (1984) Order Out of Chaos: Man's New Dialogue with Nature. Boulder, CO: New Sci. Lib. Shambhala.

6. Haken, H. (1983) Synergetics, An Introduction: Nonequilibrium Phase Transitions and Self-Organization in Physics, Chemistry, and Biology. 3rd rev. enl. ed. New York: Springer-Verlag.

7. Lasota, A. and Mackey, M. C. (1994) Chaos, Fractals and Noise: Stochastic Aspects of Dynamics. New York: Springer-Verlag

8. Abarbanel, H. D. I., Brown, R., Sidorowich, J. and Tsimring, L. (1993) The analysis of observed chaotic data in physical systems. Rev. Mod. Phys., 65, 1331-1392.

9. Tong, H. (1993) Non-Linear Time Series: A Dynamical System Approach. UK: Oxford University Press.

10. Qian, H., Shi, P.-Z. and Xing, J. (2009) Stochastic bifurcation, slow fluctuations, and bistability as an origin of biochemical complexity. Phys. Chem. Chem. Phys., 11, 4861-4870. 
11. Qian, H. (2011) Nonlinear stochastic dynamics of mesoscopic homogeneous biochemical reactions systems - an analytical theory. Nonlinearity, 24, R19-R49.

12. Wax, N. (1954) Selected Papers on Noise and Stochastic Processes. New York: Dover Pubns.

13. Onsager, L. and Machlup, S. (1953) Fluctuations and irreversible processes. Phys. Rev., 91, 1505-1512.

14. Fox, R. F. (1978) Gaussian stochastic processes in physics. Phys. Rep., 48, 179-283.

15. Ge, H. and Qian, H. (2011) Non-equilibrium phase transition in mesoscopic biochemical systems: from stochastic to nonlinear dynamics and beyond. J. R. Soc. Interface, 8, 107-116.

16. Qian, H. and Ge, H. (2012) Mesoscopic biochemical basis of isogenetic inheritance and canalization: stochasticity, nonlinearity, and emergent landscape. Mol. Cell. Biomech., 9, 1-30.

17. Monod, J. (1972) Chance and Necessity: An Essay on the Natural Philosophy of Modern Biology. New York: Vintage Books.

18. Shapiro, B. E. and Qian, H. (1997) A quantitative analysis of single protein-ligand complex separation with the atomic force microscope. Biophys. Chem., 67, 211-219.

19. Moore, P. B. (2012) How should we think about the ribosome? Annu. Rev. Biophys., 41, 1-19.

20. Phillips, R. and Quake, S. R. (2006) The biological frontier of physics. Phys. Today, 59, 38-43.

21. Bustamante, C., Liphardt, J. and Ritort, F. (2005) The nonequilibrium thermodynamics of small systems. Phys. Today, 58, 43-48.

22. Qian, H. (2012) Hill's small systems nanothermodynamics: a simple macromolecular partition problem with a statistical perspective. J. Biol. Phys., 38, 201-207.

23. Westerhoff, H. V. and Palsson, B. Ø. (2004) The evolution of molecular biology into systems biology. Nat. Biotechnol., 22, 1249-1252.

24. Qian, H. (2012) Cooperativity in cellular biochemical processes: noiseenhanced sensitivity, fluctuating enzyme, bistability with nonlinear feedback, and other mechanisms for sigmoidal responses. Annu. Rev. Biophys., 41, 179-204.

25. Beard, D. A. and Kushmerick, M. J. (2009) Strong inference for systems biology. PLoS Comput. Biol., 5, e1000459.

26. Koonin, E. V. (2009) Darwinian evolution in the light of genomics. Nucleic Acids Res., 37, 1011-1034.

27. Alberts, B. (1998) The cell as a collection of protein machines: preparing the next generation of molecular biologists. Cell, 92, 291294.

28. Elowitz, M. B., Levine, A. J., Siggia, E. D. and Swain, P. S. (2002) Stochastic gene expression in a single cell. Science, 297, 11831186.

29. Cai, L., Friedman, N. and Xie, X. S. (2006) Stochastic protein expression in individual cells at the single molecule level. Nature, 440 , 358-362.
30. Kirschner, M. W. and Gerhart, J. C. (2005) The Plausibility of Life: Resolving Darwin's Dilemma. New Haven, CT: Yale University Press.

31. Ge, H. and Qian, H. (2010) Physical origins of entropy production, free energy dissipation, and their mathematical representations. Phys. Rev. E, 81, 051133.

32. Zhang, X.-J., Qian, H. and Qian, M. (2012) Stochastic theory of nonequilibrium steady states and its applications (Part I). Phys. Rep., $510,1-86$.

33. Ge, H., Qian, M. and Qian, H. (2012) Stochastic theory of nonequilibrium steady states (Part II): Applications in chemical biophysics. Phys. Rep., 510, 87-118.

34. Jiang, D.-Q., Qian, M. and Qian, M.-P. (2004) Mathematical Theory of Nonequilibrium Steady States: On the Frontier of Probability and Dynamical Systems (Lecture Notes in Mathematics, Vol. 1833). Berlin: Springer-Verlag.

35. Von Bertalanffy, L. (1950) The theory of open systems in physics and biology. Science, 111, 23-29.

36. Qian, H. (2007) Phosphorylation energy hypothesis: open chemical systems and their biological functions. Annu. Rev. Phys. Chem., 58, $113-142$.

37. Wang, J., Xu, L. and Wang, E. K. (2008) Potential landscape and flux framework of nonequilibrium networks: robustness, dissipation, and coherence of biochemical oscillations. Proc. Natl. Acad. Sci. USA, 105, 12271-12276.

38. Wang, J., Zhang, K. and Wang, E. K. (2010) Kinetic paths, time scale, and underlying landscapes: a path integral framework to study global natures of nonequilibrium systems and networks. J. Chem. Phys., 133, 125103.

39. Wang, J., Zhang, K., Xu, L. and Wang, E. K. (2011) Quantifying the Waddington landscape and biological paths for development and differentiation. Proc. Natl. Acad. Sci. USA, 108, 8257-8262.

40. Ge, H. and Qian, H. (2012) Analytical mechanics in stochastic dynamics: most probable path, large-deviation rate function and Hamilton-Jacobi equation. Int. J. Mod. Phys. B, 26, 1230012.

41. Hanahan, D. and Weinberg, R. A. (2000) The hallmarks of cancer. Cell, $100,57-70$.

42. Ao, P., Galas, D., Hood, L. and Zhu, X.-M. (2008) Cancer as robust intrinsic state of endogenous molecular-cellular network shaped by evolution. Med. Hypotheses, 70, 678-684.

43. Ewens, W. J. (2004) Mathematical Population Genetics I. Theoretical Introduction. New York: Springer.

44. Ao, P. (2005) Laws in Darwinian evolutionary theory. Phys. Life Rev., $2,117-156$

45. Ao, P. (2008) Emerging of stochastic dynamical equalities and steady state thermodynamics from Darwinian dynamics. Commun. Theor. Phys., 49, 1073-1090.

46. Qian H. (2012) A decomposition of irreversible diffusion processes without detailed balance. arXiv.org/abs/1204.6496. 